\title{
WAS IST EIN GENERALBAß-SATZ?
}

\section{Hellmut Fed e r h of e r (Mainz)}

Dem Terminus Generalbaß-Satz räumt das Brockhaus-RiemannMusiklexikon einen eigenen Sachartikel (unmittelbar nach Generalbaß) ein. ${ }^{1}$ Er behandelt nicht das Generalbaßspiel, sondern ein Satzprinzip, das "durch eine doppelte Bezogenheit der Einzelstimme gekennzeichnet" sei. Einerseits bleibe der Kontrapunkt als Satzlehre zwar intakt, andererseits seien Besonderheiten der Stimmführung "nicht mehr vom Kontrapunkt her zu erklären, sondern ihr Bezugspol ist die Klangfolge". Daher müsse der Generalbaß-Satz - neben Kontrapunkt und Harmonielehre - »als ein Satzprinzip mit eigener Gesetzmäßigkeit verstanden werden «, ${ }^{2}$ was dazu berechtige, den Terminus mit solcher Bedeutung auszustatten und dementsprechend auch als neuen Begriff in ein Musiklexikon aufzunehmen. Verfasser des betreffenden, namentlich nicht gezeichneten Artikels ist offensichtlich $\mathrm{H}$. Haack, in dessen Dissertation über L. da Viadana nicht nur dieselben Gedanken, sondern z. T. auch wörtlich gleichlautende Formulierungen begegnen. ${ }^{3}$ Außer dieser Arbeit werden als Literaturangaben ein Zeitschriften-Aufsatz von $\mathrm{H}$. $\mathrm{H}$. Eggebrecht und Veröffentlichungen in Buchform von Th. G. Georgiades, auf den dieser neue Begriff zurückgeht, und W. Heimann angeführt. ${ }^{4}$

Georgiades versteht unter Generalbaß-Satz einen dem stile recitativo und concerto übergeordneten satztechnischen Begriff in Hinblick auf die Instrumentalisierung des Sinnzusammenhanges in der Musik J. S. Bachs. ${ }^{5}$ Bei Haack und Heimann dagegen erscheint er auf konkrete

1 Brockhaus-Riemann-Musiklexikon in 2 Bänden, hrsg. von C. Dahlhaus und H. H. Eggebrecht 1 (Wiesbaden-Mainz 1978), S. 461.

2 Ebenda.

$3 \mathrm{H}$. Haack, Anfänge des Generalbaß-Satzes I = Münchner Veröffentlichungen zur Musikgeschichte 22 (Tutzing 1974), S. 251.

4 H. H. Eggebrecht, Arten des Generalbasses im frühen und mittleren 17. Jahrhundert, in: AfMw 14 (1957), S. 61 ff. - Th. G. Georgiades, Musik und Sprache. Das Werden der abendländischen Musik, dargestellt an der Vertonung der Messe = Verständliche Wissenschaft 50 (Berlin-GöttingenHeidelberg 1954). - W. Heimann, Der Generalbaß-Satz und seine Rolle in Bachs Choral-Satz $=$ Freiburger Schriften zur Musikwissenschaft 5 (München 1973).

5 Georgiades, wie Anm. 4, S. 78 f. 
satztechnische Sachverhalte - einerseits in Kirchenkonzerten L. da Viadanas, andererseits im Choralsatz J. S. Bachs - bezogen und darüber hinaus als etablierter Begriff im Titel der betreffenden Veröffentlichungen. Daß dieser neue Terminus nicht in den Generalbaßschulen begegnet, spricht nicht gegen seine Einführung. Sollten sich mit ihm übersehene oder fälschlich gedeutete satztechnische Sachverhalte korrekter und deutlicher - als bisher - erfassen lassen?

Bekanntlich bezeichnet $\mathrm{H}$. Riemann die Epoche der Barockmusik als Generalbaß-Zeitalter. Von daher scheint es sinnvoll zu sein, das vom Generalbaß abhängige Satzgefüge als Generalbaßsatz zu benennen. $\mathrm{Da}$ sich im raschen Wandel der abendländischen Mehrstimmigkeit zwischen 1600 und 1750 das Satzbild wesentlich verändert, muß der neue Terminus jedoch sehr verschiedenartigen Satztechniken als Oberbegriff dienen. Er leidet daher - ebenso wie das Etikett Generalbaß-Zeitalter an Unverbindlichkeit. Hinzu kommt als terminologisches Bedenken, daß der Generalbaß - sofern ihm nicht nur eine dienende Funktion der Begleitpraxis zugebilligt wird - zwar Ausgangspunkt einer Satzlehre sein kann, deren Ziel aber die Beherrschung der Komposition, nicht jedoch allein die Ausführung des Generalbasses ist. Nur von einem geschaffenen Werk, d. h. vom Ergebnis einer Komposition, lassen sich strukturelle Merkmale in ihrer Gesamtheit ablesen. Sie können hingegen auch dort gleicher Art sein, wo der Generalbaß als solcher überhaupt fehlt, wie etwa in Toccaten, Präludien und anderen Werken für Tasteninstrumente. Nicht jeder $\mathrm{Ba}$ in barocker Musik ist zugleich ein Generalbaß, obwohl beider Funktion dieselbe sein kann. Diese Ansicht teilt wohl auch Haack, wenn er verallgemeinernd definiert: "Das Ergebnis der um 1600 auf der Ebene der Komposition stattfindenden Aufspaltung des bis dahin homogenen. aus Einzelstimmen grundsätzlich gleicher Faktur bestehenden Vokalsatzes in vordergründige Einzelstimmen und einem im Hintergrund veriaufenden Klangfluß nenne ich Generalbaßsatz«.6 Gewiß revolutioniert der im 17. Jahrhundert neue »Klangflu $\beta$ « das Satzgefüge, was Chr. Bernhard im Rahmen einer Stimmführungslehre zu begründen versucht. ${ }^{7}$ Aber wenig zweckmäßig erscheint es, auch dort von einem Generalbaßsatz sprechen $\mathrm{zu}$ wollen, wo ein Generalbaß als solcher gar nicht vorliegt, was aber obiger Definition zufolge erforderlich wäre.

Den sachlichen Grund für die Einführung dieses neuen Begriffs bildet zweifellos die Erkenntnis, daß sich Barockmusik - unabhängig von zeit- und örtlichen sowie gattungsmäßigen Unterschieden - weder allein durch Kategorien des Kontrapunkts noch der Harmonielehre satztechnisch befriedigend erfassen und beschreiben läßt. Allerdings liegt einer solchen Erkenntnis eine ungeprüfte Voraussetzung $\mathrm{zu}$

${ }^{6}$ Haack, wie Anm. 3, S. 52 f.

7 J. M. Müller-Blattau, Die Kompositionslehre Heinrich Schützens in der Fassung seines Schülers Christoph Bernhard (Leipzig 1926, Kassel etc. 2/1963). 
Grunde. Die im neuzeitlichen Theorieunterricht geläufige Unterscheidung beider Disziplinen verfestigt sich in ihrer Bezogenheit auf historische Sachverhalte, nämlich zur Annahme, daß der Musik v or 1600 allein eine Stimmführungslehre, jener $\mathrm{n}$ a $\mathrm{ch} 1750$ eine Lehre der Akkordfunktionen angemessen sei, zwischen welchen daher der Generalbaßsatz als Kategorie eigenen Rechts steht, so verschiedenartige satztechnische Phänomene unter ihm auch zu begreifen seien. ${ }^{8}$ Unleugbar findet diese Meinung eine gewisse Stütze im Wandel, den die Generalbaßlehre unter dem Einfluß der Harmonielehre J. Ph. Rameaus erfährt. Selbst J. Ph. Kirnberger als vermeintlich unverdächtiger Kronzeuge für die Grundsätze J. S. Bachs in satztechnischen Fragen ist - trotz seiner Differenzen mit F. W. Marpurg - ebenso wie dieser von ihr abhängig. Auch in Kirnbergers Generalbaßlehre treten an die Stelle der Baßlinie als Bezugspol des Satzes von der Kadenzlogik abstrahierte Akkordfolgen mit konsonierendem Dreiklang und »wesentlichem " Septimenakkord als Grundakkorde und Träger der Melodie. Der Septimenakkord wiederum führt zur Unterscheidung von »wesentlicher " und »zufälliger « Dissonanz, einem Unterschied, den die ältere, intervallisch regulierte Generalbaßlehre nicht kennt. Überzeugend weist Heimann nach, wie fremd eine solche Anschauungsweise J. S. Bach war, dessen »antirameauisch [e] Grundsätze« C. Ph. E. Bach bezeugt.9 Obwohl Kirnberger dieses Zeugnis von Bachs Sohn »als Argument gegen Marpurg gewertet haben möchte, so ist doch unverkennbar, daß dieser Satz seine Lehre von den beiden Grundakkorden [sc. Dreiklang und Septimenakkord] vielmehr betrifft, als daß er sie. legitimierte.$^{10}$

Bekanntlich gibt es in der Barockzeit - entsprechend der Unterscheidung stylus antiquus - stylus modernus - als Grundlage der Komposition zwei Arten musiktheoretischen Unterrichts. So heißt es in einem teils A. Bertali, teils G. Carissimi zugeschriebenen Leitfaden: "Compositio est duplex, regularis et irregularis". Während erstere »ohne Basso continuo oder beyhilff einer accompagiamenth für sich selber kan gemacht werden ", wird die compositio irregularis »erst durch den undtergezogenen Bassum continuum rectificiert «.11 Die Unentbehrlichkeit des Basses zur Rechtfertigung satztechnischer Freiheiten und die grundsätzliche Bedeutung der compositio regularis, unter der nichts anderes als der stylus antiquus gemeint ist, für die

8 z. B. Haack, wie Anm. 3, S. 69: "Ebenso wie der Generalbaßsatz hinsichtlich seiner Satztechnik historisch zwischen der Kontrapunktlehre und der Harmonielehre steht - er baut auf dem Kontrapunkt auf und bereitet die Harmonielehre vor - so steht er auch hinsichtlich der Gliederung zwischen 'zwei Kulturen der Musik' (A. Halm)«.

${ }^{9}$ Heimann, wie Anm. 4, S. 16.

10 Ebenda.

11 Nebst Quellenangabe mitgeteilt bei H. Federhofer, Zur handschriftlichen Überlieferung der Musiktheorie in Österreich in der zweiten Hälfte des 17. Jahrhunderts, in: Mf 11 (1958), S. 274. 
generalbaßbezogene compositio irregularis beweist die Bemerkung, daß zwar letztere »dieser Zeit wegen ihrer Facilitet mehrer gebrauchig", die erstere aber als Lehrgegenstand sumb ein beßers Fundament zu bekommen tauglicher ${ }^{12}$ sei. Diese Meinung vertritt auch J. J. Fux, der in den Schlußkapiteln seines Gradus ad Parnassum gleichwohl den stylus modernus seiner Zeit miteinbezieht.13 Aber nicht nur im katholischen Süden, sondern auch im protestantischen Norden wird die innere Einheit beider Stile frühzeitig erkannt. Chr. Bernhard führt die durch den Basso continuo gerechtfertigten Dissonanzfiguren des stylus modernus (communis et theatralis) mittels einer von ihm offenbar ohne Vorbild konzipierten Reduktionstechnik konsequent auf satztechnische Konstanten des stylus gravis zurück. ${ }^{14}$ Damit wird die grundsätzliche Übereinstimmung der Stimmführung in beiden Stilarten dokumentiert. Jedoch verwandelt der Fundamentcharakter des Basses im stylus modernus das lineare, intervallisch geordnete Stimmengewebe in einen generalbaß-bezogenen Akkordsatz, der eine größere melodische Beweglichkeit ermöglicht. Die wirkliche oder latente Präsenz von Akkorden gestattet eine Vermischung, Vermehrung oder Verminderung der im stylus antiquus getrennten Stimmbahnen und rechtfertigt zugleich wie Bernhard aufzeigt - auch das Ab- und Anspringen von Dissonanzen.

J. S. Bach beginnt den Kompositionsunterricht nach dem Zeugnis von C. Ph. Emanuel mit dem reinen vierstimmigen Generalbaß, um dann entsprechend den praktischen Erfordernissen eines protestantischen Kirchenmusikers zur mehrstimmigen Bearbeitung von Chorälen überzugehen. Er setzte »erstlich selbst den $B a \beta$ dazu $u$. den Alt $u$. den Tenor musten sie [sc. die Schüler] selbst erfinden. Alsden lehrte er sie selbst Bäße machen.$^{15} \mathrm{Er}$ befleißigt sich demnach der zweiten obengenannten Lehrart, welche die Stimmführung auf der Grundlage eines gegebenen, die tonale Einheit verbürgenden Basses entwickelt. Es erscheint daher legitim, die von Rameaus Konstruktion der basse fondamentale noch unbeeinflußte, bzw. unbeeinflußt gebliebene Generalbaßlehre samt den Dissonanzfiguren der zeitgenössischen Theoretiker als Instrument der Analyse Bachscher Werke heranzuziehen. ${ }^{16}$ Hingegen sind Kategorien der die Einheit des Basses auflösenden Funktionstheorie ihrer Analyse unangemessen. Allerdings sollte die Abkehr von einer einseitigen vertikalen Hör- und Betrach-

12 Ebenda.

13 Ebenda, S 277.

14 Federhofer, Der strenge und freie Satz und sein Verhältnis zur Kompositionslehre von Heinrich Schütz in der Fassung seines Schülers Christoph Bernhard, in: Ders., Beiträge zur musikalischen Gestaltanalyse (Graz-Innsbruck-Wien 1950), S. 61 ff.

15 C. Ph. E. Bachs 2. Brief an J. N. Forkel, in: Veröffentlichungen der Neuen Bachgesellschaft, Jg. 17, H. 3, hrsg. von M. Schneider. Hier zitiert nach Heimann, wie Anm. 4, S. 131.

16 Heimann, a. a. O., S. 130 ff. 
tungsweise nicht dazu führen, sich mit dem zu begnügen, was mit der zeitgenössischen Theorie zu leisten imstande ist. Zwar werden durch Generalbaßbezifferung die Klangfolgen unterschiedslos nach intervallischen Aspekt beurteilt. Daraus folgt jedoch keine "praktizierte intervallische Denkweise ${ }^{17}$ unter Ausschluß der Harmonie. Bachs Kontrapunkt läßt sich allein als Intervallsatz nicht befriedigend deuten, da er durch seine baßmäßige Fundierung Anteil am Akkord hat. Die Alternative: baßbezogener Intervalisatz - harmonischfunktioneller Akkordsatz erweist sich als musiktheoretische Fiktion und geht an der Realität vorbei, weil einerseits der den Oberstimmen gemeinsame Baßbezug bereits die Harmonie impliziert, andererseits der Funktionsbegriff $\mathrm{zu}$ eng gefaßt erscheint, wenn er allein die Folge von Akkord zu Akkord betreffen soll und aus diesem zu Unrecht verengten Blickwinkel als der Bachschen Harmonik nicht konform auf Ablehnung stößt.

Hier ist der Anlaß, auf Stimmführungsanalysen von Werken J. S. Bachs durch $\mathrm{H}$. Schenker hinzuweisen, dessen Musiktheorie bekanntlich dem Verhältnis der Stimmführung zur Harmonik als zentralem Problem der Tonalität gewidmet ist. Schenkers Satzanalyse des Chorals $\mathrm{Nr} 16$ Ich bin's, ich sollte büssen der Matthäuspassion kann das Gemeinte verdeutlichen. ${ }^{18}$ Beide, bis auf den Schluß melodisch identischen Choralzeilen stattet Bach mit verschiedenem $\mathrm{Baß}$ und voneinander abweichenden Mittelstimmen aus. Die erste Zeile schließt mit einem Quintteiler - der V. Stufe bzw. Dominante der schulmäßigen Harmonielehre -, die zweite mit einem Ganzschluß auf der Tonika. Die melodisch identischen Klauseln in den Takten 2 und 8, bzw. 4 und 10 nehmen durch die verschiedene Baßführung einen jeweils anderen harmonischen Stellenwert ein. Es fragt sich nun, in welcher Weise der Außenstimmensatz, der das substantielle Gefüge darstellt, satztechnisch zu verstehen ist. Stellt er nur ein Summenereignis intervallisch und stimmführungsmäßig korrekter Fortschreitungen von Klausel zu Klausel dar oder verkörpert er selbst eine tonale Idee?

Untersucht man zuerst den Verlauf des Basses, so zeigt sich, daß er in den ersten Choralzeilen abwärtsschreitend den Tonikaklang horizontal aufrollt und dann mit dem Grundton des Quintteilers schließt:

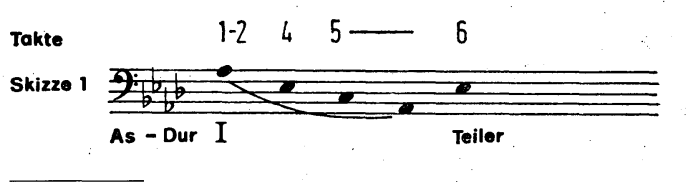

17 Ebenda, S. 18.

18 H. Schenker, Fünf Urlinie-Tafeln (Wien 1932). - Ders., Der Freie Satz = Neue musikalische Theorien und Phantasien 3 (Wien 1935), Anhang: Figurentafeln, S. III. 
In der zweiten Choralzeile schreitet der $\mathrm{Ba}$ chromatisch von es über $e \mathrm{zu} f$ weiter, das in den Takten 7-11 als Kernton festgehalten wird. Die häufige Wiederkehr dieses Tons als Grundton des f-mollbzw. F-Dur-Klangs (Takte 7, 8, 10 und 11) auf guten bzw. relativ guten Taktteilen und die melodische Bewegung, die sich dazwischen im $\mathrm{Ba} \beta$ und in den anderen Stimmen vollzieht, berechtigen dazu, dem Ton $f$ diese strukturelle Bedeutung beizumessen: $b$ in Takt 8 und $c$ in Takt 10 sind nur Unter- und Oberquinte von $f$, zu dem beide im Sprung wieder zurückkehren. Folgende Skizze diene zur Verdeutlichung:

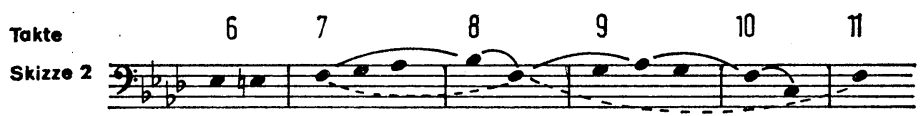

Erst im vorletzten Takt wird as als Träger der Tonika wieder erreicht. Die metrische Stellung dieses $A s$-Dur-Dreiklanges bewirkt seine Gewichtsabstufung gegenüber jenem in Takt 9, zweites Viertel, der primär als Stimmführungsereignis zu werten ist, weil er innerhalb der Auskomponierung des f-moll-Dreiklangs steht. Der Baß verläuft daher in der zweiten Choralzeile vom Quintteiler es in Takt 6 ausgehend, stufenweise aufwärts bis $\mathrm{zu}$ as in Takt 11 (vgl. den Bogen in der folgenden Skizze), das zu des, es, As als Träger der Stufen IV V- I weiterschreitet:

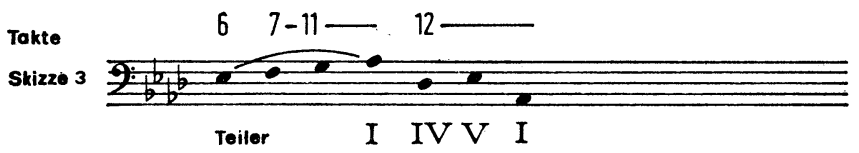

Die in beiden Zeilen bis auf den abweichenden Schluß identische, in der Oberstimme liegende Choralmelodie umschreibt gleich $\mathrm{zu} \mathrm{Be-}$ ginn mit $c^{2}-a s^{1}$ jenen Tonraum, in dem das Gerüst der Melodie zu suchen ist. In der ersten Zeile schreitet sie von $c^{2}$ nach $b^{1}$ (Takt 6), in der zweiten von $c^{2}$ über $b^{1}$ nach $a s^{1}$ (Takt 12). Schenker spricht von einem fallenden Terzzug, der am Schluß der ersten Zeile eine Unterbrechung durch den Quintteiler erfährt und erst in der zweiten zu Ende geführt wird. Diese, den Gesamtverlauf bestimmende fallende Strukturterz, die nachfolgende Skizze durch Balken zwischen den betreffenden Noten hervorhebt, wird durch eine Reihe teils fallender, teils steigender Terzen untergliedert. Letztere werden durch Klammern angedeutet: 


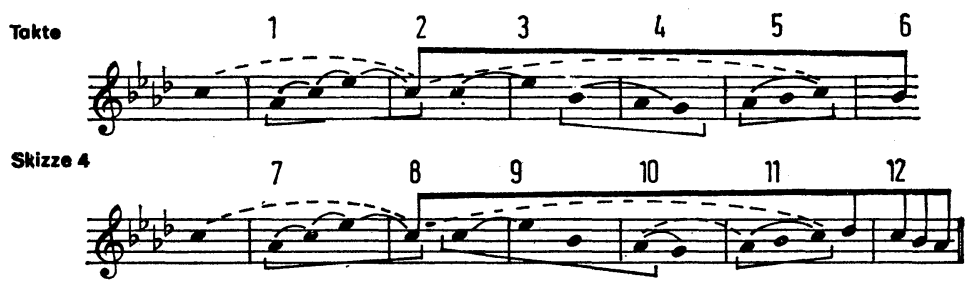

Faßt man beide Außenstimmen mit den Mittelstimmen zusammen - wie dies Schenker getan hat ${ }^{19}$ - so zeigt sich, daß sie gemeinsam die Idee einer horizontalen Entfaltung des $A s$-Dur-Klangs verwirklichen. Er ist daher als Tonikaklang zu bezeichnen, und in seiner zeitlichen Verwirklichung liegt der tonale Sinn dieses Choralsatzes beschlossen.

Ist also $\mathrm{zu}$ Recht die Funktionstheorie, die sich unter Außerachtlassung der Stimmführung und der Baßgestalt auf die funktionelle Deutung von Akkordfolgen beschränkt, als Erklärungsprinzip des Bachschen Choralsatzes abzulehnen, so doch nicht der Nachweis einer durch die Stimmführung verwirklichten tonräumlichen Ordnung, an der sowohl die horizontale, als auch die vertikale Dimension ihren Anteil haben. Eine solche Betrachtungsweise impliziert einen Tonalitätsbegriff, der sich nicht allein aus der Kadenz ableitet, sondern die Stimmführung miteinschließt. Er setzt ein schichtenmäßig differenziertes Stufenbewußtsein voraus, das sich als hierarchische Anordnung der Akkorde erst aus der Mitberücksichtigung der Stimmführung ergibt. ${ }^{20}$

Ansätze hierzu zeigt gewiß auch die Klangtechnik Viadanas. Aber es dürfte unmöglich sein, dessen Generalbaßkonzerte ähnlich wie den Choralsatz J.S. Bachs als horizontale Entfaltung eines einzigen Klanges zu verstehen. Ihnen liegt ein anderer Tonalitätstypus zu Grunde, in dem die verschiedenen satztechnischen Phänomene (gliedernde Wirkung der Kadenz, häufiges Vorkommen quintverwandter Klänge, konstruktive Tonfolgen in Baß, Freiheit der Oberstimme und Supplementstimmen) ${ }^{21}$

19 Ebenda. Die einzige Abweichung in obigen Skizzen 2 und 4 von Schenkers Analyse betrifft die Deutung des $f$-Klanges in Takt 10. Schenker betrachtet ihn als Durchgang innerhalb von Terzzügen in den Außenstimmen, wohl um eine mit den Takten 3-4 übereinstimmende strukturelle Deutung der Oberstimme zu ermöglichen, was jedoch wegen der metrisch starken Position der $f$-Klanges in Takt 10 fragwürdig erscheint. Hingegen wird ihm in den obigen Skizzen 2 und 4 schon jene Bedeutung zugebilligt, die Schenker erst dem $f$-Klang im folgenden Takt 11 beimißt. Der C-Klang in Takt 10 ist Oberquintteiler des $f$-Klanges.

20 Federhofer, Akkord und Stimmführung in den musiktheoretischen Systemen von H. Riemann, E. Kurth und H. Schenker, in:Sitzungsberichte der Österreichischen Akademie der Wissenschaften, phil.-hist. Klasse 380 (Wien 1981).

${ }_{21}$ Haack, wie Anm. 4, S. 251. 
die ihnen an und für sich zukommende Bedeutung noch nicht einem Prinzip unterordnen, das den Satzverlauf als Weg von der Tonika zur Dominante und wieder zurück zur Tonika ausweist. Insofern weicht Viadanas Satz nicht nur in Einzelheiten, sondern prinzipiell von jenem Bachs ab. Die satztechnischen Unterschiede entsprechen generell jenen zwischen Früh- und Spätbarock. Diese Erkenntnis der Verschiedenheit scheint den Verfasser des einleitend zitierten Lexikonartikels auch zur Feststellung bewogen $\mathrm{zu}$ haben, daß der Generalbaß-Satz »nicht (wie Kontrapunkt und Harmonielehre) als Satzlehre greifbar ist ${ }^{22}$. Diese Feststellung ist gewiß richtig. Aber gerade deshalb sollte dieser Terminus - ebenso wie aus ähnlichen Gründen der Terminus Generalbaß-Harmonik, den schon C. Dahlhaus für fragwürdig hält, ${ }^{23}$ in der kritisierten Bedeutung besser vermieden werden.

\section{POVZETEK}

Osređnje razmišljanje velja vprašanju, ali je uporaba izraza kompozicijski stavek $\mathrm{z}$ generalnim basom (Generalbaß-Satz) sploh umestna. Tega pojmuje namreč $\mathrm{H}$. Haack kot poseben kompozicijski princip z lastno zakonitostjo, kot rezultat razcepitve vokalnega stavka - ki je bil do okrog leta 1600 homogen in je obstajal iz posameznih partov načelno enake fakture - $\mathrm{v}$ izstopajoče glasove in zvočni tok, ki poteka $\mathrm{v}$ ozadju. Prav. v zvezi s takšnim pojmovanjem izraža avtor vrsto pomislekov in prihaja do sklepa, da kompozicijski stavek $\mathrm{z}$ generalnim basom ni tako konkretno oprijemljiv kot nauk o kontrapunktu ali harmoniji in se potemtakem kaže podobno vprašljiv kot harmonija $\mathrm{z}$ generalnim basom (Generalbaß-Harmonie). Glede na omenjeno definicijo bi pravzaprav bilo treba govoriti o stavku z generalnim basom tudi $\mathrm{v}$ tistih primerih, ko tega sploh ni, tako pri kompozicijah za instrumente s tipkami, kar pa je vse prej kot smiselno. Strukturalne značilnosti dela je treba razbrati iz dela kot celote, te značilnosti pa so lahko enake tudi tedaj, ko generalni bas manjka. Ne sme se pozabiti, da so že skladatelji in teoretiki baroka videli neko notranjo enovitost obeh tedanjih kompozicijskih načinov, ki ju predstavljata stylus antiquus in stylus modernus. Končno se ne sme prezreti dejstva, da se kompozicijski stavek J. S. Bacha načeloma razlikuje od tistega L. Viadane, čigar temelj je drugačen tip tonalitete, pri čemer gre torej za kompozicijsko-tehnične razlike, ki v splošnem ustrezajo razlikam med zgodnjim in poznim barokom.

${ }_{22}$ Brockhaus-Riemann-Musiklexikon, wie Anm. 1, S. 461.

23 C. Dahlhaus, Untersuchungen über die Entstehung der harmonischen Tonalität $=$ Saarbrücker Studien zur Musikwissenschaft 2 (Kassel etc. 1968), S. 125. 